\title{
Role of RAS mutation status as a prognostic factor for patients with advanced colorectal cancer treated with first-line chemotherapy based on fluoropyrimidines and oxaliplatin, with or without bevavizumab: A retrospective analysis
}

\author{
JAVIER GARDE NOGUERA ${ }^{1}$, ELOISA JANTUS-LEWINTRE ${ }^{2}$, MIREIA GIL-RAGA $^{3}$, ELENA EVGENYEVA $^{4}$, \\ SONIA MACIÁ ESCALANTE ${ }^{5}$, ANTONIO LLOMBART-CUSSAC ${ }^{1}$ and CARLOS CAMPS HERRERO ${ }^{6}$ \\ ${ }^{1}$ Medical Oncology Department, Hospital Arnau de Vilanova of Valencia, 46015 Valencia; ${ }^{2}$ Molecular Oncology Laboratory, \\ University General Hospital of Valencia, Research Foundation, 46014 Valencia; ${ }^{3}$ Medical Oncology Department, \\ Hospital de Sagunto, 46520 Valencia; ${ }^{4}$ Pathology Department, Hospital Marina-Salud de Denia, 03700 Dénia; \\ ${ }^{5}$ Medical Oncology Department, Hospital General de Elda, 03600 Elda; ${ }^{6}$ Medical Oncology and Molecular Laboratory \\ Department, University General Hospital of Valencia, University of Valencia, 46014 Valencia, Spain
}

Received January 29, 2016; Accepted December 2, 2016

DOI: $10.3892 / \mathrm{mco} .2017 .1149$

\begin{abstract}
The role of Kirsten rat sarcoma viral oncogene homolog (KRAS) and neuroblastoma RAS viral oncogene homolog (NRAS) mutations as negative predictors for anti-epidermal growth factor receptor (EGFR) therapies in metastatic colorectal cancer (CRC) has been firmly established. However, whether the RAS mutation status plays a role as a biomarker for anti-vascular endothelial growth factor (VEGF) treatment remains controversial. Data from 93 CRC patients who received first-line cytotoxic chemotherapy with fluoropyrimidines and oxaliplatin, with or without bevacizumab, were analyzed. We investigated the association between the RAS mutation status and clinical outcomes in terms of response rate, progression-free survival (PFS) and overall survival (OS). Mutations in RAS genes were observed in $47(52.6 \%)$ patients (45 KRAS and 2 NRAS mutations). Patients with tumours harbouring RAS mutations were less suitable for primary tumour resection, were more likely to develop lung metastases, and received bevacizumab treatment for a shorter time period compared with those with wild-type tumours. The response rate to chemotherapy did not differ according to the RAS mutation status, and there were no significant differences in PFS [RAS mutation: 12 months, 95\% confidence interval (CI): 8.7-15.2 vs. RAS wild-type: 12 months, 95\% CI: 9.67-14.32; $\mathrm{P}=0.857$ ] or OS (RAS
\end{abstract}

Correspondence to: Dr Javier Garde Noguera, Medical Oncology Department, Hospital Arnau de Vilanova of Valencia, 12 San Clemente Street, 46015 Valencia, Spain

E-mail: javiergardenoguera1@gmail.com

Key words: RAS mutation, prognostic factor, bevacizumab, colorectal cancer mutation: 20 months, 95\% CI: $14.3-25.6$ vs. RAS wild-type: 24 months, 95\% CI: 18.7-29.2; $\mathrm{P}=0.631$ ). Patients with RAS mutation vs. those with RAS wild-type exhibited a favourable trend in PFS when treated with bevacizumab (13 months, 95\% CI: $6.5-19.4$ vs. 10 months, 95\% CI: 4.2-15.7, respectively; $\mathrm{P}=0.07)$ and $\mathrm{OS}(27$ months, 95\% CI: $18.5-35.4$ vs. 15 months, 95\% CI: $12.4-17.5$, respectively; $\mathrm{P}=0.22$ ). In conclusion, RAS mutations are not a prognostic marker for PFS and OS in CRC patients receiving fluoropyrimidine-oxaliplatine treatment, with or without bevacizumab. RAS mutations are not predictive of the lack of efficacy of bevacizumab, and these patients appear to benefit from anti-angiogenic treatment.

\section{Introduction}

In Western countries, $20 \%$ of patients with colorectal cancer (CRC) present with advanced disease stage at diagnosis (1-3). For $>40$ years, the standard treatment approach for advanced CRC with inoperable metastasis has been systemic chemotherapy. With the advances in systemic chemotherapy for metastatic CRC, survival has increased from 12 months with 5-fluorouracil monotherapy to $\sim 2$ years with the addition of oxaliplatin, irinotecan and targeted or biological agents (4-6). There is a need for identifying biomarkers for these biological agents that lead to a personalized approach to cancer treatment, ensuring maximum efficacy while simultaneously minimizing toxicity and treatment-related side effects.

Vascular endothelial growth factor (VEGF) and epithelial growth factor receptor (EGFR) are involved in molecular pathways related to the growth, survival, proliferation and metastasis of tumour cells. Targeted agents that are able to inhibit signal transduction through these proteins have been incorporated into the standard first-line treatment of advanced CRC $(7,8)$. Anti-EGFR therapies with the monoclonal antibodies cetuximab and panitumumab improve chemotherapeutic efficacy, but this effect is restricted to patients with wild-type Kirsten 
rat sarcoma viral oncogene homolog (KRAS) and neuroblastoma RAS viral oncogene homolog (NRAS) mutations $(9,10)$. Thus, assessing the RAS status is currently a routine procedure worldwide to identify patients that would not benefit from such treatment in order to avoid unnecessary toxicity. However, bevacizumab, a monoclonal antibody against VEGF, was the first inhibitor of angiogenesis approved for the treatment of advanced CRC in combination with chemotherapy, based on the survival benefit observed in clinical trials. However, no biomarker able to identify patients who may benefit from this therapy has been established to date (7).

KRAS is a proto-oncogene encoding a small $21-\mathrm{kD}$ guanosine triphosphate/guanosine diphosphate-binding protein involved in the regulation of cellular response to several extracellular stimuli (11). Mutations within KRAS abrogating GTPase activity and resulting in activation of RAS/RAF signaling are found in 35-42\% of CRCs and are considered to occur early during CRC carcinogenesis. Activating mutations in other members of the RAS oncogene family (HRAS and NRAS) have also been described, although they are significantly less frequent. NRAS appears in $2 \%$ of patients with advanced CRC (12). The presence of NRAS mutations has also recently been associated with a lack of benefit from anti-EGFR therapies (13), and has been incorporated in clinical practice as a predictive biomarker to select first-line treatment for patients with advanced CRC.

VEGF is an important regulator of physiological as well as pathological angiogenesis, and it is overexpressed in a number of different tumour types. It has been demonstrated that RAS pathway signaling increases VEGF expression and represses negative regulators of angiogenesis, suggesting that RAS aberrations may modulate the tumour response to anti-angiogenic therapies (14-16). It remains controversial whether KRAS mutation, independently of the use of anti-EGFR therapies, has a prognostic role in CRC $(17,18)$. Different studies published to date have not been conclusive, even several large studies $(19,20)$, and the role of KRAS and NRAS mutation status as a predictor of outcome of oxaliplatin-based chemotherapy and bevacizumab remains uncertain.

The aim of this study was to evaluate the role of RAS mutation status as a predictive and prognostic factor in patients with advanced CRC treated with first-line standard chemotherapy with fluoropyrimidines and oxaliplatin, with or without bevacizumab.

\section{Patients and methods}

Study design and ethics. This was a multicenter and retrospective study of patients presenting with advanced CRC at diagnosis who were treated with fluoropyrimidine and oxaliplatin combination chemotherapy regimens, with or without bevacizumab, at four different hospitals in Valencia, Spain.

The study was performed following approval by the Independent Ethics Committees of the participating institutions and in accordance with the Declaration of Helsinki, the Good Clinical Practices and local ethical and legal requirements (Spanish laws). Prior to study entry, all the patients (or their relatives) provided written informed consent according to the local ethics committee regulations. This study complied with all applicable regulations for human participant studies.
Patient characteristics. The medical records of patients diagnosed with advanced CRC were reviewed. The patients had pathologically confirmed advanced colorectal adenocarcinoma available for evaluation of KRAS and NRAS mutations, and had been treated between January, 2009 and December, 2012 with a first-line chemotherapy regimen involving FOLFOX or XELOX, with or without bevacizumab.

Treatment and follow-up. All enrolled patients were treated with fluoropyrimidine and oxaliplatin combination chemotherapy regimens, with or without bevacizumab. A complete review of the medical history and baseline measures of the tumour prior to treatment initiation were performed to evaluate the patients. The diagnosis and treatment evaluation were performed with computed axial tomography.

The following data were collected from inpatient and outpatient records: Relevant clinical data, such as age, gender, presence of symptoms related to the tumour (weight loss, haemorrhage or bowel occlusion), comorbidities and Eastern Cooperative Oncology Group performance statuand tumour-specific data, such as location of primary metastases, number of organs with metastatic involvement and resection of primary tumour. Finally, data on therapy (chemotherapeutic regimen, overall response); time-to-disease progression and overall survival were collected.

Treatment toxicity was assessed according to the criteria published by the National Cancer Institute Common Toxicity Criteria, version 2.0 (https://ctep.cancer.gov/protocolDevelopment/electronic_applications/docs/ctcmanual_v4_10-4-99. pdf). Tumour response was evaluated according to Response Evaluation Criteria In Solid Tumours, version 1.1 (21). OS was calculated as the time from diagnosis to the date of death, and PFS as the time from treatment initiation to the documented date of disease progression.

RAS mutation analysis. The analysis of RAS mutations was performed using the TheraScreen ${ }^{\circledR}$ KRAS Pyro kit (for KRAS codons 12, 13 and 61), and the TheraScreen ${ }^{\circledR}$ RAS Extension Pyro kit (for KRAS codons 59, 117 and 146, and NRAS codons 12, 13, 59, 61, 117 and 146) (Qiagen, Madrid, Spain), according to the manufacturer's instructions. Briefly, $5 \mu \mathrm{l}$ of template DNA (10 ng of genomic DNA) were amplified by polymerase chain reaction (PCR) using, $12.5 \mu \mathrm{l}$ of PyroMark ${ }^{\circledR}$ PCR Master Mix 2x, $2.5 \mu \mathrm{l}$ of Coral Load Concentrate 10x, $4 \mu \mathrm{l}$ of nuclease-free water, and $1 \mu \mathrm{l}$ of the corresponding set of PCR primers (Qiagen). The reactions took place in a MasterCycler ${ }^{\circledR}$ thermocycler (Eppendorf, Hamburg, Germany) under the following cycling conditions: $94^{\circ} \mathrm{C}$ for $15 \mathrm{~min}$; 42 cycles of denaturation at $95^{\circ} \mathrm{C}$ for $20 \mathrm{sec}$; annealing at $53^{\circ} \mathrm{C}$ for $30 \mathrm{sec}$, followed by extension at $72^{\circ} \mathrm{C}$ for $20 \mathrm{sec}$.

The amplicons were immobilised on Streptavidin Sepharose ${ }^{\circledR}$ High Performance beads (Qiagen) to prepare the single-stranded DNA and the sequencing primers were annealed to it using a PyroMark Q24 plate and a vacuum workstation (Qiagen). PyroMark Gold Q24 reagents (enzyme mixture, substrate mixture and nucleotideall from Qiagen) were then prepared and loaded into a cartridge so they could be dispensed during the sequencing process. Finally, the plate and the cartridge were loaded into the PyroMark Q24 System and the sequencing process was initiated. The sequences were 
analysed using software provided by the manufacturer(Qiagen). In each run, two controls were included: Unmethylated control DNA, which worked as a positive control for PCR and sequencing reactions, and a negative control (without template DNA).

Statistical analysis. All statistical analyses were performed using the SPSS statistical package, version 16 (SPSS, Inc., Chicago, IL, USA). A descriptive statistics analysis, including measures of central tendencies and dispersions of quantitative variables, as well as absolute and relative frequencies for categorical variables, was also performed; t-test was used to compare two independent samples of continuous variables.

The Chi-square test was used to compare two or more independent groups of subjects with respect to a given categorical variable. PFS and OS according to KRAS status were analyzed using the Kaplan-Meier method to estimate the probability of survival and survival difference with the use of the log-rank test. All reported P-values were the result of two-sided tests, with $\mathrm{P}<0.05$ considered statistically significant.

\section{Results}

Patient characteristics. A total of 93 patients with advanced CRC and available samples for NRAS and KRAS analysis, who were treated with fluoropyrimidine and oxaliplatin combination chemotherapy regimens (XELOX or FOLFOX), with or without bevacizumab, were identified. A total of 49 patients $(52.6 \%)$ had tumours with RAS mutations, namely 47 KRAS and 2 NRAS mutations. The patient characteristics (of the global population and by RAS status) are summarized in Table I. The median age of the patients was 65 years (range, 39-83 years) at diagnosis, and the male/female ratio was $1.5 / 1.0$. The characteristics of the patients were generally similar between the RAS mutation and wild-type groups. The proportion of patients with RAS mutations who underwent surgery for the primary tumour was significantly lower compared with that of patients with RAS wild-type tumours $(\mathrm{P}=0.019)$, and they exhibited a higher rate of lung metastases (34.6 vs. 15.9\%, respectively; $\mathrm{P}=0.03$ ). Furthermore, patients with RAS mutations were less likely to receive bevacizumab in the first-line treatment setting compared with the wild-type population (29.5 vs. $44-8 \%$, respectively; $\mathrm{P}=0.09$ ).

Treatment efficacy. The overall response rate (ORR) with first-line chemotherapy treatment was $53.8 \%$, and the disease control rate (DCR) was $81.2 \%$. There were no significant differences in ORR according to the RAS mutation status (mutation vs. wild-type, 48.9 vs. $58.9 \%$, respectively; $\mathrm{P}=0.129$ ), as shown in Table II.

The median PFS for the global population was 12 months, without significant differences between groups [RAS mutation: 12 months, 95\% confidence interval (CI): 8.7-15.2 vs. RAS wild-type: 12 months, 95\% CI: 9.67-14.32; $\mathrm{P}=0.857]$. The median OS was 22 months, without a significant difference according to the RAS status (RAS mutation: 20 months, 95\% CI: 14.3-25.6 vs. RAS wild-type: 24 months, $95 \%$ CI: 18.7-29.2; $\mathrm{P}=0.631$ ), as shown in Fig. 1 .
Patients treated with bevacizumab exhibited a median PFS of 12 vs. 11 months for those treated with chemotherapy alone $(\mathrm{P}=0.055)$. Significant differences in OS according to the use of bevacizumab in the overall population were not observed, although there was a favourable trend for patients treated with the combination of chemotherapy and bevacizumab (patients receiving bevacizumab reached an OS of 27 months $(95 \% \mathrm{CI}$ : 21.9-32), whereas patients without bevacizumab reached an OS of 20 months (95\% CI: 13.8-26.1; $\mathrm{P}=0.25)$. Patients with RAS mutations also exhibited a non-significant favourable trend in PFS when treated with bevacizumab (13 months, 95\% CI: 6.5-19.4) compared with those treated with chemotherapy alone (10 months, 95\% CI: 4.2-15.7; $\mathrm{P}=0.07)$. The median OS was longer in patients with RAS mutations who received bevacizumab, but this difference did not reach statistical significance (27 months, 95\% CI: $18.5-35.4$ vs. 15 months, 95\% CI: 12.4-17.5, respectively; $\mathrm{P}=0.22$ ) as shown in Fig. 2. Furthermore, the median OS of patients treated with bevacizumab was similar between the RAS mutation and wild-type groups (27.0 vs. 27.0 months, respectively; $\mathrm{P}=0.562$ ).

\section{Discussion}

This retrospective study was designed to analyze the prognostic role of RAS mutations in patients with advanced CRC treated with fluoropyrimidine and oxaliplatin chemotherapy, with or without bevacizumab. We also assessed whether patients with RAS mutations obtain any benefit from bevacizumab treatment.

The predictive and prognostic value of KRAS mutations in patients with advanced CRC treated with first-line chemotherapy and anti-EGFR therapy has been confirmed by retrospective analysis of phase III trials with cetuximab and panitumumab $(9,10)$. Amado et al (9) published data from a randomized trial comparing panitumumab monotherapy with best supportive care (BSC) in patients with chemotherapy-refractory advanced CRthey detected KRAS mutations in $43 \%$ of the patients, and observed that the efficacy of panitumumab was significantly higher in the wild-type group in terms of PFS (12.3 vs. 7.3 weeks, respectively; $\mathrm{P}<0.001$ ), response rate (17 vs. $0 \%$, respectively) and OS [hazard ratio $(\mathrm{HR})=0.67$; 95\% CI: 0.55-0.82]. Similarly, Karapetis et al (10) reported results from 394 patients included in a phase III trial that compared cetuximab with BSC in chemotherapy-refractory advanced CRthey detected KRAS mutations in $42.3 \%$ of the patients, and the presence of this molecular aberration was associated with a lack of benefit from cetuximab treatment in terms of OS (4.8 vs. 9.5 months, respectively; $\mathrm{HR}=0.55$; 95\% $\mathrm{CI}$ : $0.41-0.74$; $\mathrm{P}<0.001)$ and $\mathrm{PFS}$ (1.9 vs. 3.78 months, $\mathrm{HR}=0.40 ; 95 \% \mathrm{CI}$ : $0.30-0.54 ; \mathrm{P}<0.001$ ). They also observed that the mutation status of the KRAS gene did not affect survival among patients treated with BSC alone. More recently, mutations in NRAS, another member of the RAS oncogene family, that appear in $2-5 \%$ of patients with advanced $\mathrm{CRC}$, have also been found to predict lack of response to anti-EGFR treatment (22). Doudillard et al retrospectively analyzed the efficacy and safety of panitumumab plus FOLFOX chemotherapy according to the RAS (KRAS and NRAS) mutation status (22); they detected NRAS mutations in $3.4 \%$ of the patients, and their association with 
Table I. Clinicopathological factors.

\begin{tabular}{|c|c|c|c|c|}
\hline Variables & Total $(\mathrm{n}=93)$ & RAS wild-type $(n=44)$ & RAS mutation $(\mathrm{n}=49)$ & P-value \\
\hline Age, years (range) & $65(39-83)$ & $68.5(46-80)$ & $68(39-83)$ & \\
\hline Gender & & & & 0.101 \\
\hline Male & $56(60.2)$ & $30(68.1)$ & $26(53.0)$ & \\
\hline Female & $37(39.8)$ & $14(31.9)$ & $23(47.0)$ & \\
\hline Performance status, n (\%) & & & & 0.402 \\
\hline $0-1$ & $76(81.1)$ & $35(79.5)$ & $41(83.7)$ & \\
\hline 2 & $17(19.9)$ & $9(20.1)$ & $8(16.3)$ & \\
\hline \multicolumn{5}{|c|}{ Tumour-related symptoms, n (\%) } \\
\hline Weight loss $>10 \%$ & $17(18.3)$ & $10(22.7)$ & $7(17.0)$ & 0.143 \\
\hline Bleeding & $30(32.3)$ & $16(47.0)$ & $7(17.0)$ & 0.184 \\
\hline Occlussion & $8(8.6)$ & $1(3.0)$ & $14(34.1)$ & 0.056 \\
\hline \multicolumn{5}{|c|}{ Surgery of primary tumour, n (\%) } \\
\hline Yes & $49(52.6)$ & $18(41.0)$ & $16(32.6)$ & $0.019^{\mathrm{a}}$ \\
\hline No & $42(47.4)$ & $26(59.0)$ & $33(67.3)$ & \\
\hline \multicolumn{5}{|l|}{ Location of metastases, n (\%) } \\
\hline Liver & $75(80.6)$ & $38(86.3)$ & $37(75.5)$ & 0.145 \\
\hline Peritoneum & $19(20.4)$ & $10(22.7)$ & $9(18.3)$ & 0.396 \\
\hline Lung & $24(25.8)$ & $7(15.9)$ & $17(34.6)$ & $0.033^{\mathrm{a}}$ \\
\hline Bone & $3(3.2)$ & $0(0.0)$ & $3(6.1)$ & 0.142 \\
\hline Lymph node & $22(23.7)$ & $10(22.7)$ & $12(24.4)$ & 0.519 \\
\hline \multicolumn{5}{|c|}{ Number of metastatic locations, n (\%) } \\
\hline 1 & $52(55.9)$ & $25(56.8)$ & $27(20.4)$ & 0.517 \\
\hline$\geq 2$ & $48(44.1)$ & $19(43.1)$ & $22(44.9)$ & \\
\hline \multicolumn{5}{|l|}{$\begin{array}{l}\text { Operable metastases after } \\
\text { chemotherapy, n (\%) }\end{array}$} \\
\hline Yes & $17(19.8)$ & $10(25.0)$ & $7(15.2)$ & 0.194 \\
\hline No & $69(80.0)$ & $30(75.0)$ & $39(84.7)$ & \\
\hline \multicolumn{5}{|l|}{ Serum levels, n (\%) } \\
\hline CEA (high) & $62(66.7)$ & $30(75.0)$ & $32(76.1)$ & 0.552 \\
\hline LDH (high) & $32(52.5)$ & $15(53.5)$ & $17(51.5)$ & 0.539 \\
\hline Haemoglobin (low) & $22(28.6)$ & $10(27.7)$ & $12(29.2)$ & 0.544 \\
\hline \multicolumn{5}{|c|}{ Grade of differentiation, $\mathrm{n}(\%)$} \\
\hline 1 & $12(12.9)$ & $5(15.6)$ & $7(21.2)$ & 0.693 \\
\hline 2 & $43(46.2)$ & $22(68.7)$ & $21(63.6)$ & \\
\hline 3 & $9(9.7)$ & $5(15.6)$ & $4(12.1)$ & \\
\hline \multicolumn{5}{|c|}{ Chemotherapy scheme, n (\%) } \\
\hline FOLFOX/XELOX & $58(62.4)$ & $13(29.5)$ & $22(44.8)$ & 0.095 \\
\hline FOLFOX/XELOX-B & $35(37.6)$ & $31(70.5)$ & $27(55.1)$ & \\
\hline
\end{tabular}

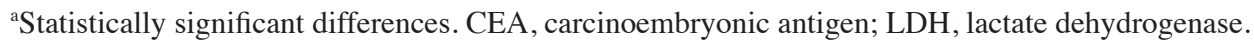

a shorter PFS (10.1 vs. 7.9 months, respectively; $\mathrm{HR}=0.72$; 95\% CI: $0.58-0.90 ; \mathrm{P}=0.004)$ and $\mathrm{OS}(26.0$ vs. 20.2 months, respectively; $\mathrm{HR}=0.78 ; 95 \% \mathrm{CI}: 0.62-0.99 ; \mathrm{P}=0.04)$. Thus, the presence of RAS mutations is considered to be a negative predictive factor of response to anti-EGFR therapies. However, its role as a prognostic factor for OS in patients treated with chemotherapy alone, or in combination with bevacizumab, remains unclear.
The data reported by our study suggest that RAS status does not have a prognostic value for PFS or OS in patients with advanced CRC treated with optimal first-line chemotherapy. Our study, similar to other studies published to date investigating this issue, has a retrospective nature and may yield conflicting results. Our findings are in accordance with data from other small retrospective studies that did not identify an association between RAS mutation status and patient 
Table II. Response to treatment, n (\%).

\begin{tabular}{lccc}
\hline Response & RAS mutation & RAS wild-type & P-value \\
\hline Complete response & $0(0.0)$ & $2(4.5)$ & 0.129 \\
Partial response & $24(48.9)$ & $24(54.4)$ & \\
Stable disease & $12(24.4)$ & $7(15.9)$ & \\
Progressive disease & $10(20.4)$ & $6(13.6)$ & \\
\hline
\end{tabular}

A

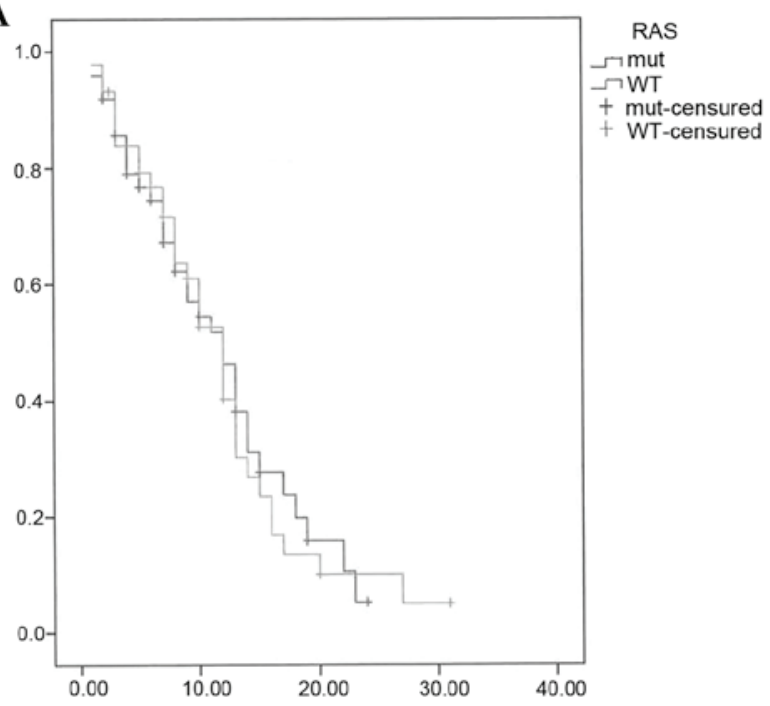

B

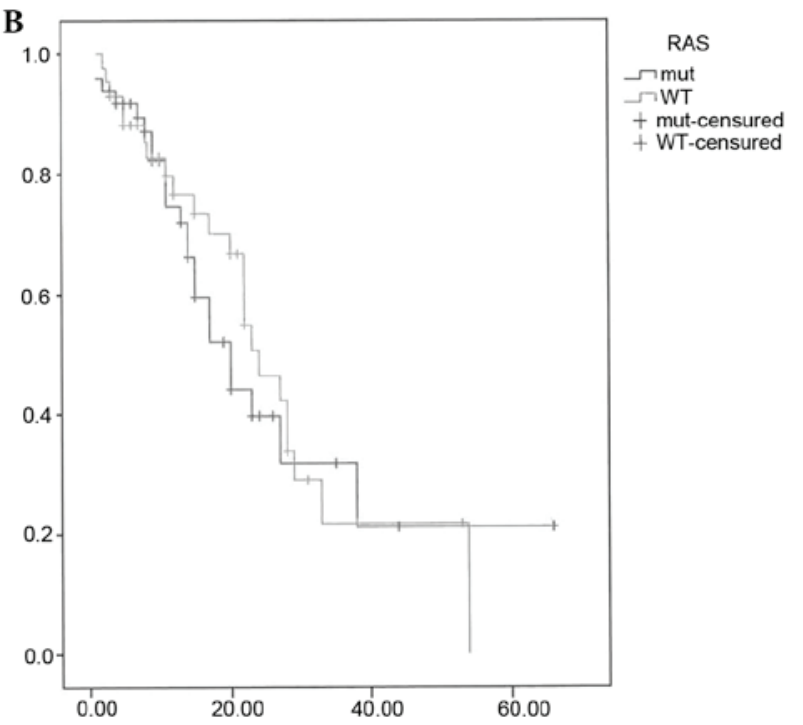

Figure 1. (A) Progression-free survival (PFS) and (B) overall survival (OS) by RAS status. WT, wild-type; mut, mutation.

outcome (23-25). Kim et al analyzed 103 patients evaluable for KRAS mutation status treated with chemotherapy without anti-EGFR therapy, and they did not observe differences in response rate, PFS or OS according to RAS status (25). Two large collaborative studies of the KRAS by the Colorectal Cancer Collaborative Group (RASCAL) reported conflicting results $(26,27)$. While the first RASCAL study reported an increased risk of recurrence and mortality associated with KRAS mutations, the second study refined this observation
A

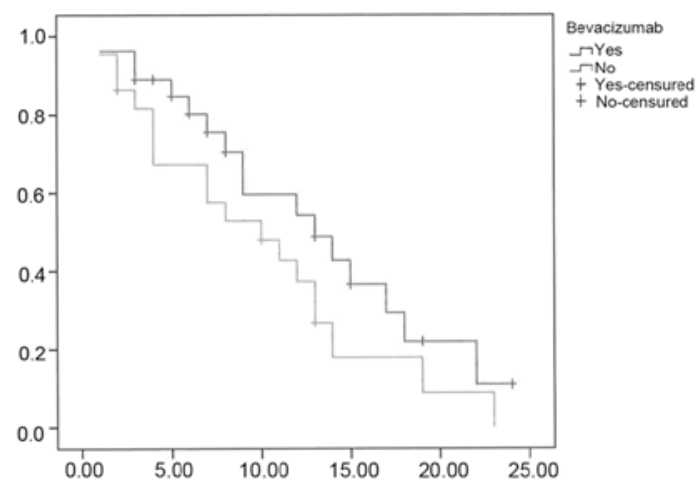

B

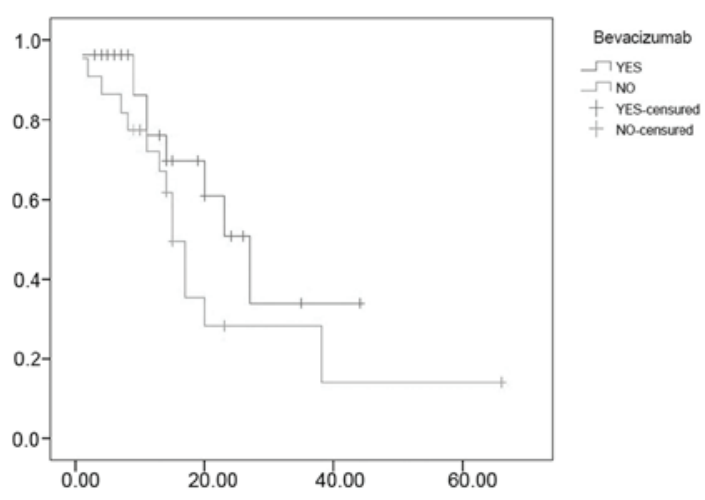

Figure 2. (A) Progression-free survival (PFS) and (B) overall survival (OS) of patients with tumours harbouring RAS mutations according to treatment with bevacizumab.

to report a significant prognostic value in failure-free survival only with the G12V mutation in Dukes' C patients. It is difficult to compare the results of all these retrospective studies, since there are several confounding factors that may affect the findings. The majority of these series are based on small patient samples, and none of the previously published studies incorporated in their analysis the presence of NRAS mutationhowever, it is remarkable that most conclude that there is no association between RAS mutation status and patient outcome.

Regarding our second objective, we observed no association between RAS mutation status and the efficacy of bevacizumab. We observed that patients with RAS mutation presented with a longer median PFS and OS when treated with bevacizumab; however, the difference was not statistically significant. Furthermore, we observed no difference in PFS or OS in patients treated with bevacizumab according to the RAS status. Our findings suggest that RAS mutational status has no predictive value for bevacizumab outcome in patients with advanced CRC. Our findings are consistent with previous retrospective studies. Hurwitz et al observed no apparent association between the improved PFS and KRAS status for patients treated with bevacizumab and irinotecan and fluoropyrimidine chemotherapy (28). More recently, the MAX study confirmed that KRAS mutation status was neither prognostic for OS nor predictive for bevacizumab outcome in patients with advanced CRC (29). These two studies analyzed the role of KRAS mutations in patients treated with irinotecan-or mytomicin-based chemotherapy plus bevacizumab, which are not the most commonly used chemotherapeutic regimens worldwide in the first-line setting. Kim et al (30) published a 
retrospective study of 32 patients with advanced CRC treated with bevacizumab and cytotoxic chemotherapy based on oxaliplatin; they observed that the KRAS mutation status was neither predictive for bevacizumab nor a prognostic factor for OS in these patients. All our patients received standard cytotoxic oxaliplatin and fluoropyrimidine treatment, with or without bevacizumab, and were analyzed for NRAS and KRAS mutations, adding significant evidence on the lack of effect of RAS mutation status on the outcome of bevacizumab.

In summary, these findings suggest that RAS mutation status is not a prognostic factor for OS in patients with advanced CRC, and is also not predictive of the response to bevacizumab in combination with an optimal chemotherapy regimen based on oxaliplatin and fluoropyrimidines.

\section{References}

1. National Cancer Institute: Surveillance, Epidemiology and End Results Program. www.seer.cancer.gov. Accessed May, 2013.

2. Edge SB, Byrd DR, Compton CC, Fritz AG, Greene FL and Trotti A (Eds): AJCC cancer staging manual. 7th edition. Springer, New York, NY, p143, 2010.

3. Siegel R, Naishadham D and Jemal A: Cancer statistics, 2012. CA Cancer J Clin 62: 10-29, 2012.

4. Bleiberg H: Role of chemotherapy for advanced colorrectal cancer. New oportunities. Semin Oncol 23 (Suppl 3): 42-50, 1996.

5. de Gramont A, Louvet C, André T, Tournigand C and Krulik M: A review of GERCOD trials of bimonthly leucovorin plus 5-fluorouracil 48-h continuous infusion in advanced colorectal cancer: Evolution of a regimen. Groupe d'Etude et de Recherche sur les Cancers de l'Ovaire et Digestifs (GERCOD). Eur J Cancer 34: 619-626, 1998.

6. Capdevila J, Elez E, Peralta S, Macarulla T, Ramos FJ and Tabernero J: Oxaliplatin-based chemotherapy in the management of colorectal cancer. Expert Rev Anticancer Ther 8: 1223-1236, 2008.

7. McCormack PL and Keam SJ: Bevacizumab: A review of its use in metastatic colorectal cancer. Drugs 68: 487-506, 2008.

8. Blick SK and Scott LJ: Cetuximab: A review of its use in squamous cell carcinoma of the head and neck and metastatic colorectal cancer. Drugs 67: 2585-2607, 2007.

9. Amado RG, Wolf M, Peeters M, Van Cutsem E, Siena S, Freeman DJ, Juan T, Sikorski R, Suggs S, Radinsky R, et al: Wild-type KRAS is required for panitumumab efficacy in patients with metastatic colorectal cancer. J Clin Oncol 26 : 1626-1634, 2008.

10. Karapetis CS, Khambata-Ford S, Jonker DJ, O'Callaghan CJ, Tu D, Tebbutt NC, Simes RJ, Chalchal H, Shapiro JD, Robitaille $\mathrm{S}$, et al: K-ras mutations and benefit from cetuximab in advanced colorectal cancer. N Engl J Med 359: 1757-1765, 2008.

11. Schubbert S, Shannon K and Bollag G: Hyperactive Ras in developmental disorders and cancer. Nat Rev Cancer 7: 295-308, 2007.

12. Irahara N, Baba Y, Nosho K, Shima K, Yan L, Dias-Santagata D, Iafrate AJ, Fuchs CS, Haigis KM and Ogino S: NRAS mutations are rare in colorrectal cancer. Diagn Mol Pathol 19: 157-163, 2010.

13. De Roock W, Claes B, Bernasconi D, De Schutter J, Biesmans B, Fountzilas G, Kalogeras KT, Kotoula V, Papamichael D, Laurent-Puig P, et al: Effects of KRAS, BRAF, NRAS, and PIK3Ca mutations on the efficacy of cetuximab plus chemotherapy in chemotherapy-refractory metastatic colorrectal cancer: A retrospective consortium analisis. Lancet Oncol 11: 753-762, 2010.

14. Chin L, Tam A, Pomerantz J, Wong M, Holash J, Bardeesy N, Shen Q, O'Hagan R, Pantginis J, Zhou H, et al: Essential role for oncogenic Ras in tumour maintenance. Nature 400: 468-472, 1999.
15. Rak J, Mitsuhashi Y, Bayko L, Filmus J, Shirasawa S, Sasazuki T and Kerbel RS: Mutant ras oncogenes upregulate VEGF/VPF expression: Implications for induction and inhibition of tumor angiogenesis. Cancer Res 55: 4575-4580, 1995.

16. Watnick RS, Cheng YN, Rangarajan A, Ince TA and Weinberg RA: Ras modulates Myc activity to repress thrombospondin-1 expression and increase tumor angiogenesis. Cancer Cell 3: 219-231, 2003.

17. Klump B, Nehls O, Okech T, Hsieh CJ, Gaco V, Gittinger FS, Sarbia M, Borchard F, Greschniok A, Gruenagel HH, et al: Molecular lesions in colorectal cancer: Impact on prognosis? Original data and review of the literature. Int J Colorectal Dis 19: 23-42, 2004.

18. Roth AD, Tejpar S, Delorenzi M, Yan P, Fiocca R, Klingbiel D, Dietrich D, Biesmans B, Bodoky G, Barone C, et al: Prognostic role of KRAS and BRAF in stage II and III resected colon cancer: Results of the translational study on the PETACC-3, EORTC 40993, SAKK 60-00 trial. J Clin Oncol 28: 466-474, 2010.

19. Samowitz WS, Curtin K, Schaffer D, Robertson M, Leppert M and Slattery ML: Relationship of Ki-ras mutations in colon cancers to tumor location, stage, and survival: A population-based study. Cancer Epidemiol Biomarkers Prev 9: 1193-1197, 2000.

20. Ince WL, Jubb AM, Holden SN, Holmgren EB, Tobin P, Sridhar M, Hurwitz HI, Kabbinavar F, Novotny WF, Hillan KJ and Koeppen H: Association of k-ras, b-raf, and p53 status with the treatment effect of bevacizumab. J Natl Cancer Inst 97: 981-989, 2005.

21. Eisenhauer EA, Therasse P, Bogaerts J, Schwartz LH, Sargent D, Ford R, Dancey J, Arbuck S, Gwyther S, Mooney M, et al: New response evaluation criteria in solid tumours: Revised RECIST guideline (version 1.1). Eur J Cancer 45: 228-247, 2009.

22. Doudillard JY, Oliner KS, Siena S, Tabernero J, Burkes R, Barugel M, Humblet Y, Bodoky G, Cunningham D, Jassem J, et al: Panitumumab-FOLFOX4 treatment and RAS mutations in colorrectal cancer. N Engl J Med 369: 1023-1034, 2013.

23. González-Aguilera JJ, Oliart S, Azcoita MM and Fernández-Peralta AM: Simultaneous mutations in K-ras and TP53 are indicative of poor prognosis in sporadic colorectal cancer. Am J Clin Oncol 27: 39-45, 2004.

24. Westra JL, Schaapveld M, Hollema H, de Boer JP, Kraak MM, de Jong D, ter Elst A, Mulder NH, Buys CH, Hofstra RM and Plukker JT: Determination of TP53 mutation is more relevant than microsatellite instability status for the prediction of disease-free survival in adjuvant-treated stage III colon cancer patients. J Clin Oncol 23: 5635-5643, 2005.

25. Kim ST, Park KH, Kim JS, Shin SW and Kim YH: Impact of KRAS mutation status on otucome in metastatic colon cancer patients without anti-epidermal growth factor receptor therapy. Cancer Res Treat 45: 55-62, 2013.

26. Andreyev HJ, Norman AR, Cunningham D, Oates JR and Clarke PA: Kirsten ras mutations in patients with colorectal cancer: The multicenter 'RASCAL' study. J Natl Cancer Inst 90: 675-684, 1998

27. Andreyev HJ, Norman AR, Cunningham D, Oates J, Dix BR, Iacopetta BJ, Young J, Walsh T, Ward R, Hawkins N, et al: Kirsten ras mutations in patients with colorectal cancer: The 'RASCAL II' study. Br J Cancer 85: 692-696, 2001.

28. Hurwitz H, Fehrenbacher L, Novotny W, Cartwright T, Hainsworth J, Heim W, Berlin J, Baron A, Griffing S, Holmgren E, et al: Bevacizumab plus irinotecan, fluorouracil, and leucovorin for metastatic colorectal cancer. N Engl J Med 350: 2335-2342, 2004.

29. Price TJ, Hardingham JE, Lee CK, Weickhardt A, Townsend AR, Wrin JW, Chua A, Shivasami A, Cummins MM, Murone C and Tebbutt NC: Impact of KRAS and BRAF gene mutation status on outcomes from the Phase III AGITG MAX Trial of capecitabine alone or in Combination with bevacizumab and mitomycin in advanced colorectal cancer. J Clin Oncol 29: 2675-2682, 2011.

30. Kim ST, Park KH, Shin SW and Kim YH: Does KRAS mutation status affect on the effect of VEGF therapy in metastatic colon cancer patients? Cancer Res Treat 46: 48-54, 2014. 